\title{
Adjuvant therapy in type 1 diabetes mellitus
}

\author{
Edyta Cichocka ${ }^{\circledR}$, Anna Maj-Podsiadło, Janusz Gumprecht \\ Department of Internal Medicine, Diabetology, and Nephrology, Faculty of Medical Sciences in Zabrze, Medical University of \\ Silesia, Katowice, Poland
}

Key words: type 1 diabetes mellitus; adjuvant therapy

The introduction of new groups of hypoglycaemic drugs has been associated with a growing interest in adjuvant therapy in type 1 diabetes (T1DM). The aim of such treatment is to support exogenous insulin therapy to achieve the therapeutic goal and at the same time to reduce the risk of hypoglycaemia and to have a beneficial effect on body weight. Potential therapeutic options include metformin (which has been used for many years) and new hypoglycaemic drugs, such as SGLT2 inhibitors and GLP-1 analogues. This article shows three female patients who were successfully treated with adjuvant therapy due to poor glycaemic control of T1DM and comorbidities.

\section{Patient 1 - SGLT2 inhibitor}

A 42-year-old obese female patient (BMI $40.2 \mathrm{~kg} / \mathrm{m}^{2}$ ) with a 21-year history of T1DM presented at a follow-up visit. The patient had been treated with an insulin pump for 6 years (60 IU; Aspart). Non-compliance with dietary recommendations, consumption of additional snacks without insulin administration, and a gradual weight gain of $8 \mathrm{~kg}$ over four years was noted $\left(\mathrm{HbA}_{1 \mathrm{c}}-8.4 \%\right)$. Additionally, the patient had a history of dyslipidaemia and hypothyroidism (levothyroxine $100 \mathrm{ug} /$ day $-\mathrm{TSH}$ $-1.8 \mathrm{IU} / \mathrm{mL}$ ). The patient was offered therapy with dapagliflozin $5 \mathrm{mg} /$ day due to poor glycaemic control and after excluding contraindications to such treatment (pregnancy test, urinalysis, biochemical tests). The patient was instructed about the possibility of reducing insulin requirements in the initial period of treatment, the risk of hypoglycaemia and ketoacidosis, and was also recommended strict glycaemic control and monitoring of ketone bodies in the urine. She was also instructed about the principles of proper hygiene to prevent urogenital infections and was reminded about the rules of proper nutrition and the necessity of increasing physical activity. After three months, during the follow-up visit, a weight loss of $3.5 \mathrm{~kg}$ was observed, the glycaemic control was improved $\left(\mathrm{HbA}_{1 \mathrm{c}}-7.6 \%\right)$, and the mean glycaemic level based on the data from the insulin pump was $135 \pm 43 \mathrm{mg} / \mathrm{dL}$. No adverse effects of the SGLT2 inhibitor were reported by the patient. She finally managed to lose weight. No changes in insulin requirements were noted. The dose of dapagliflozin was increased to $10 \mathrm{mg}$. Other recommendations were maintained. A reduction in the body weight of $6 \mathrm{~kg}$ was observed. Furthermore, improvement in glycaemic control was also achieved $\left(\mathrm{HbA}_{1 \mathrm{c}}-7.1 \%\right)$.

\section{Patient 2-GLP-1 analogue}

A 39-year-old obese female patient with a 15-year history of T1DM complicated by diabetic retinopathy after laser treatment of the right eye reported to the Outpatient Clinic of Diabetology for a follow-up visit. For 2 years, the patient had been treated with an insulin pump (Paradigm G640) with a continuous glucose monitoring (CGM) system (64 IU/day; Lispro). In addition, the patient presented with a history of hypothyroidism in autoimmune thyroiditis (levothyroxine $25 \mathrm{ug} /$ day) and hypertension (torasemide $5 \mathrm{mg} /$ day). The patient had gained weight (20 $\mathrm{kg}$ ) following the second delivery, and her weight at the follow-up visit was $106 \mathrm{~kg}\left(\mathrm{BMI}-38 \mathrm{~kg} / \mathrm{m}^{2}\right)$. She reported difficulties in following dietary recommendations and the impossibility to implement physical activity due to numerous duties (2 young children, professional work). Prior to the visit, $\mathrm{HbA}_{1 \mathrm{c}}$ was $7.2 \%$ and the mean glycaemic level based on the data from the insulin pump was $132 \pm 55 \mathrm{mg} / \mathrm{dl}$. Despite satisfactory glycaemic control, the patient was offered adjuvant therapy with liraglutide, which she approved. As in the case of the first patient, this patient was instructed about the aim, cost of therapy, possible adverse effects, and the necessity of effective contraception during the treatment. After performing laboratory tests and the exclusion of contraindications, liraglutide $0.6 \mathrm{mg} /$ day s.c. was started, and the patient was also instructed to increase the dose every week, depending on the drug tolerance. Four months later, she reported for a follow-up visit. The tolerance of the treat- 
ment was good, and the dose of liraglutide was gradually increased to $1.8 \mathrm{mg} /$ day. Adverse effects such as nausea and vomiting occurred only during the first three weeks of treatment and were acceptable. Due to these adverse effects, the patient was examined to exclude diabetic ketoacidosis (normal arterial blood gas test, trace of ketone bodies in the urine). Pregnancy was excluded. $\mathrm{HbA}_{1 \mathrm{c}}$ was $6.6 \%$ and the mean glycaemic level based on the data from the insulin pump was $122 \pm 26 \mathrm{mg} / \mathrm{dl}$. A decrease in insulin requirements (by 25\%) was observed (the daily dose was $49 \mathrm{IU})$, and better arterial pressure control was reported, which resulted in discontinuation of the hypotensive drug. A weight loss of $6 \mathrm{~kg}$ was observed, and the patient's compliance with dietary recommendations was better. A total weight loss of $11 \mathrm{~kg}$ was noted within one year following the administration of liraglutide.

\section{Patient 3 - metformin}

A 34-year-old obese female patient with a 20-year history of T1DM complicated by non-proliferative retinopathy was admitted to the Department of Diabetology to start insulin pump therapy as part of pregnancy planning. The patient had a history of diabetes mellitus, which was treated with multiple insulin injections (the total daily insulin dose was 115 IU). Despite a long history of the disease, she had never been trained in terms of carbohydrate counting or self-adjustment of insulin doses. On admission, $\mathrm{HbA}_{1 \mathrm{c}}$ was $9.2 \%$, and morning hyperglycaemia (up to $240 \mathrm{mg} / \mathrm{dL}$ ) was the most prevalent. Additionally, the patient had a history of hypothyroidism in autoimmune thyroiditis - treated with levothyroxine $150 \mathrm{ug} /$ day (TSH - $3.45 \mathrm{IU} / \mathrm{mL}$ ) - and polycystic ovary syndrome (PCOS; treated with combined oral contraceptive pill with cyproterone acetate). Her body weight was $86 \mathrm{~kg}$ (BMI — 31.9 $\mathrm{kg} / \mathrm{m}^{2}$ ). In addition, mixed hypercholesterolaemia was diagnosed during hospitalisation (TC $-5.6 \mathrm{mmol} / \mathrm{L}, \mathrm{TG}$ $-2.54 \mathrm{mmol} / \mathrm{L}$ ). Dietary instructions were given to the patient during hospitalisation and she was instructed to count carbohydrate and protein-fat exchanges. Insulin pump therapy was started to improve her daily glycaemic profile. Due to comorbidities (PCOS) and increased insulin resistance (insulin requirement of $1.3 \mathrm{UI} / \mathrm{kg}$ ), the dose of metformin was gradually increased $(1500 \mathrm{mg} /$ day at discharge) and the dose of levothyroxine was also increased. After three months, during the follow-up visit to the Outpatient Diabetes Clinic, the glycaemic control improved. The patient followed functional intensive insulin therapy. Based on the data from the insulin pump, the mean glycaemic level was $142 \pm 48 \mathrm{mg} / \mathrm{dL}$ and $\mathrm{HbA}_{1 \mathrm{c}}$ was $7.8 \%$. The daily insulin dose was decreased by $22 \%$ (90 IU/day). The patient's body weight was 83 $\mathrm{kg}$ (a decrease of $3 \mathrm{~kg}$ ). The follow-up concentration of TSH was $2.26 \mathrm{IU} / \mathrm{mL}$, and the lipid profile also improved (TC $-5.2 \mathrm{mmol} / \mathrm{L}, \mathrm{TG}-2.0 \mathrm{mmol} / \mathrm{L}$ ). The patient did not report any metformin-related adverse effects. The dose of metformin was gradually increased $(850 \mathrm{mg} / 3$ times a day), and the patient was recommended to reduce the dose of insulin if necessary. After 10 months of insulin pump therapy, glycaemic control was achieved and planning the pregnancy was possible $\left(\mathrm{HbA}_{1 \mathrm{c}}-6.6 \%\right.$, $\mathrm{TSH}-1.9 \mathrm{IU} / \mathrm{mL}$ ). The daily insulin dose was $75 \mathrm{IU}$ (decrease of 35\%). The patient's body weight was reduced by $10 \mathrm{~kg}$ and her weight was $76 \mathrm{~kg}$. The dose of metformin (2550 mg/d) was maintained.

Despite the development of insulin therapy and the methods for CGM in the treatment of T1DM, many patients do not achieve satisfactory glycaemic control. In addition, intensive insulin therapy has adverse effects such as weight gain and hypoglycaemia. In addition to absolute insulin deficiency, an increasing number of patients experience a whole spectrum of metabolic syndrome-related disorders, including obesity and paradoxically insulin resistance [1]. Currently, functional insulin therapy is the cornerstone of the treatment of T1DM, but adjuvant therapies are used more commonly, such as metformin [2-3], SGLT2 inhibitors [4-5], and GLP-1 analogues [6]. In conclusion, in addition to insulin, the number of therapeutic options is increasing for patients with T1DM, especially for those with concomitant obesity and metabolic syndrome. When decisions about treatment are made, the following should be considered: indications listed in the summary of product characteristics and the related limitations (off-label therapy), no reimbursement (which is related to high treatment costs), and the possibility of adverse reactions, which necessitates careful selection of patients and their appropriate education.

\section{Conflict of interest}

None declared.

\section{References}

1. Polsky S, Ellis SL. Obesity, insulin resistance, and type 1 diabetes mellitus. Curr Opin Endocrinol Diabetes Obes. 2015; 22(4): 277-282, doi: 10.1097/MED.0000000000000170, indexed in Pubmed: 26087341.

2. Al Khalifah RA, Alnhdi A, Alghar $\mathrm{H}$, et al. The effect of adding metformin to insulin therapy for type 1 diabetes mellitus children: A systematic review and meta-analysis. Pediatr Diabetes. 2017; 18(7): 664-673, doi: 10.1111/pedi.12493, indexed in Pubmed: 28145083.

3. Libman IM, Miller KM, DiMeglio LA, et al. T1D Exchange Clinic Network Metformin RCT Study Group. Effect of Metformin Added to Insulin on Glycemic Control Among Overweight/Obese Adolescents With Type 1 Diabetes: A Randomized Clinical Trial. JAMA. 2015; 314(21): 2241-2250, doi: 10.1001/jama.2015.16174, indexed in Pubmed: 26624824.

4. Maj-Podsiadło A, Cichocka E, Gumprecht J. SGLT-2 inhibitors as adjunctive to insulin therapy in type 1 diabetes. Clin Diabetol. 2020; 9(3): 189-192, doi: 10.5603/dk.2020.0013.

5. Huang Y, Jiang Z, Wei Y. Short- and medium-term efficacy of sodium glucose cotransporter 2 (SGLT-2) inhibitors for the treatment of type 1 diabetes: systematic review and meta-analysis. Endokrynol Pol. 2020; 71(4): 325-333, doi: 10.5603/EP.a2020.0034, indexed in Pubmed: 32901912.

6. Mathieu C, Zinman B, Hemmingsson JU, et al. ADJUNCT ONE Investigators. Efficacy and Safety of Liraglutide Added to Insulin Treatment in Type 1 Diabetes: The ADJUNCT ONE Treat-To-Target Randomized Trial. Diabetes Care. 2016; 39(10): 1702-1710, doi: 10.2337/dc16-0691, indexed in Pubmed: 27506222 